\title{
Prácticas sobre cuidado de niños con discapacidad construidas por cuidadores del Instituto de Capacitación Los Álamos
}

\section{Caregiving Practices for Children with Disabilities Constructed by Caregivers at the Instituto de Capacitación Los Álamos}

\section{Práticas sobre o cuidado de crianças com deficiência construídas pelos cuidadores do Instituto de Capacitación Los Álamos}

\author{
Nidia Johana Arias Becerra ${ }^{1}$, ORCID 0000-0002-4261-3310 \\ Jessica María Ayala Hernández, ${ }^{2}$, ORCID 0000-0002-3385-3355 \\ Andrea Taborda Mazo ${ }^{3}$, ORCID 0000-0003-0364-7734 \\ Andres Choperena Posada ${ }^{4}$, ORCID 0000-0003-1423-4353 \\ Alejandra González Mejía ${ }^{5}$, ORCID 0000-0002-3585-9857 \\ Juan Felipe Naranjo Lopez ${ }^{6}$, ORCID 000-0002-5093-5424 \\ María Clara Tuberquia Velásquez ${ }^{7}$, ORCID 0000-0002-5453-9875 \\ 1234567 Universidad CES, Colombia
}

\begin{abstract}
Resumen: Introducción: La labor de cuidado de niños con discapacidad en primera infancia está mediada por muchos elementos, entre ellos, las prácticas. Dada la discapacidad y la edad de los niños, es habitual que sean sus cuidadores los responsables de su bienestar. Comprender las prácticas de los cuidadores permite optimizar e implementar rutinas funcionales, que propendan al bienestar y la calidad de vida de los niños y sus cuidadores. Objetivo: Comprender las prácticas de personas encargadas del cuidado a niños con discapacidad, de 1 a 6 años de edad, en el Instituto de Capacitación Los Álamos (Itagüí, Colombia). Método: Estudio cualitativo enfocado en la teoría fundamentada. Se invitó a participar 12 madres sustitutas del instituto, se realizó un muestreo a conveniencia hasta la saturación teórica. Resultados: Las prácticas se constituyen en rutinas de cuidado que incluyen actividades que son específicas para los niños con discapacidad, tales como masajes, terapias, adaptaciones del espacio para el posicionamiento y la participación, entre otros. Actividades como la alimentación y el masaje son igual de importantes a la hora del cuidado de un niño con discapacidad. Conclusión: El cuidado de niños con discapacidad supone la realización de múltiples actividades y diferentes prácticas específicas para la condición del niño. A pesar de lo difíciles que son, terminan estando inmersas en un compilado de rutinas que se incorporan al día a día de la familia.
\end{abstract}

Palabras claves: niños con discapacidad; cuidadores; cuidado del niño; actividades cotidianas. 
Abstract: Introduction: Many elements, including practices, mediate the work of caring for children with disabilities in early childhood. As a result of the disability and age of the children, their caregivers commonly become responsible for their well-being. Understanding the practices of caregivers allows optimizing and implementing functional routines that promote the well-being and quality of life of children and their caregivers. Objective: To understand the practices of caregivers of children aged 1-6 years with disabilities at the Instituto de Capacitación Los Álamos (Itagüí, Colombia). Method: Qualitative study focused on grounded theory. The participants were twelve surrogate mothers from the institute; a convenience sampling was carried out until theoretical saturation. Results: The practices consisted of care routines, including activities specifically for children with disabilities such as massages, therapies, adaptations of the space for postural control and participation. Moreover, activities such as feeding and massages are equally important when caring for a child with a disability. Conclusion: Caring for children with disabilities involves multiple activities and practices specific to the child's condition. Despite their difficulty, they become immersed in a compilation of routines incorporated into the family's daily life.

Keywords: children with disabilities; caregivers; childcare; everyday activities.

Resumo: Introdução: O trabalho de cuidar de crianças com deficiência na primeira infância é mediado por muitos elementos, incluindo práticas. Dada a deficiência e a idade das crianças, é comum que seus cuidadores sejam responsáveis por seu bem-estar. A compreensão das práticas dos cuidadores permite otimizar e implementar rotinas funcionais que promovam o bem-estar e a qualidade de vida da criança e de seus cuidadores. Objetivo: Compreender as práticas sobre o cuidado de crianças com deficiência física na primeira infância, construídas por cuidadores pertencentes ao Instituto de Capacitación Los Álamos. Método: Estudo qualitativo com foco na teoria fundamentada. Doze mães de aluguel foram convidadas a participar, sendo realizada uma amostragem por conveniência até a saturação teórica. Resultados: As práticas se constituem em rotinas de cuidado, que incluem atividades específicas para crianças com deficiência, como massagens, terapias, adaptações do espaço para posicionamento e participação, entre outras. Atividades como alimentação e massagem são tão importantes quanto ao cuidar de uma criança com deficiência. Conclusão: Cuidar de crianças com deficiência envolve a realização de múltiplas atividades e diferentes práticas específicas à condição da criança. Por mais difíceis que sejam, acabam imersos em uma compilação de rotinas que se incorporam ao dia a dia da família.

Palavras-chave: crianças com deficiência; cuidadores; cuidado da criança; atividades cotidianas.

Recibido: 05/03/2021

Aceptado: 29/10/2021

Cómo citar:

Arias Becerra NJ, Ayala Hernández JM, Taborda Mazo A, Choperena Posada A, González Mejía A, Naranjo Lopez JF, Tuberquia Velásquez MC. Prácticas sobre cuidado de niños con discapacidad construidas por cuidadores del Instituto de Capacitación Los Álamos. Enfermería: Cuidados Humanizados. 2021;10(2):191-203. DOI: 10.22235/ech.v10i2.2485 


\section{Introducción}

Según el Informe Mundial de Discapacidad del año 2011, la discapacidad forma parte de la condición humana. Casi todas las personas sufrirán algún tipo de discapacidad transitoria o permanente en algún momento de su vida. Se estima que más de mil millones de personas viven con algún tipo de discapacidad, o sea, alrededor del $15 \%$ de la población mundial, según las estimaciones en 2010. ${ }^{(1)}$

Según la Encuesta Mundial de Salud, solo la carga mundial de morbilidad mide las discapacidades infantiles de 0 a 14 años, con una estimación de 95 millones de niños $(5,1 \%)$, 13 millones de los cuales $(0,7 \%)$ tienen "discapacidad grave", es decir, dificultades muy significativas de funcionamiento. ${ }^{(2)}$

Los niños que experimentan discapacidad desde los primeros años de vida se encuentran expuestos a factores de riesgo como la pobreza, el estigma y la discriminación, una mala interacción con sus cuidadores, la violencia, el abuso y el abandono, y el acceso limitado a programas y servicios, todo lo cual puede afectar significativamente su supervivencia y desarrollo, haciendo que pase a un segundo plano. ${ }^{(3)}$

Dado que se trata de niños con discapacidad, la protección de sus derechos se rige bajo la Convención sobre los Derechos del Niño (CDN) y la Convención sobre los Derechos de las Personas con Discapacidad (CDPD). A pesar de esto, se encuentran múltiples vulneraciones de sus derechos. Los niños que están en la primera infancia dependen en gran medida de sus cuidadores, y es en este punto donde las prácticas de los cuidadores toman un especial valor. ${ }^{(3)}$

Las prácticas de cuidado marcan el curso en la condición del niño, pudiendo mejorarla o empeorarla y con ello impactar positiva o negativamente la situación del núcleo familiar. Las prácticas relacionadas con el cuidado, entre ellas el posicionamiento, solo se evidencian cuando no se realizan adecuadamente; si se suma el factor tiempo, revertir sus consecuencias es difícil. Por lo anterior, una buena formación a los cuidadores y un empoderamiento de su rol, construido en el día a día, hace del cuidado un factor clave en la inclusión de los niños con discapacidad. ${ }^{(4)}$ Comprender la forma como se realiza el cuidado permite a los cuidadores validar sus prácticas con el equipo interdisciplinario y, con ello, generar cambios esenciales en su quehacer y el de los demás profesionales de la institución.

El objetivo de este trabajo es caracterizar las prácticas sobre cuidado de niños con discapacidad construidas por cuidadores del Instituto de Capacitación Los Álamos. Como objetivo específico, se propone identificar los tipos de práctica que se construyen alrededor del cuidado de los niños con discapacidad.

\section{Método}

Se trata de un estudio cualitativo que permitió comprender el mundo de la experiencia vivida, a partir de las prácticas como cuidadores de niños con discapacidad desde el punto de vista que las personas viven. El proceso de indagación fue inductivo y el investigador interactuó con los participantes en busca de las respuestas a preguntas que se centraron en la experiencia como cuidador. El estudio fue basado en la teoría fundamentada, donde las categorías teóricas derivadas de los datos, mediante la utilización de un método comparativo, permitió el alcance de la construcción teórica sustantiva, la teorización del cuidado y las actividades que este incluye. 
En el estudio participaron 12 mujeres, madres sustitutas a cargo de niños con discapacidad motora, con edad comprendida entre 1 y 6 años. Las participantes pertenecían a estratos socioeconómicos 2 y 3. Ocho de ellas vivían en el área urbana del área metropolitana de Medellín. Fueron contactadas a partir de la base de datos proporcionada por el Instituto de Capacitación y posteriormente invitadas a participar del estudio.

Las madres hacían parte del Instituto de Capacitación Los Álamos, operador de programas del Instituto Colombiano de Bienestar Familiar (ICBF). El término madre sustituta puede apoyarse en lo que De la Cuesta y Benjumea denominan cuidador formal, dado que reciben una remuneración económica por su labor. ${ }^{(5)}$ Se realizó un muestreo por conveniencia, que finalizó con la saturación teórica de las categorías. ${ }^{(6)}$ Ningún miembro del equipo investigador tenía una relación previa con las participantes del estudio. El estudio se realizó entre el año 2018 y 2019. El grupo investigador estuvo conformado por 5 mujeres y 2 hombres. El equipo contaba con experiencia en el planteamiento, construcción y realización del trabajo de campo en enfoques cualitativos. Asimismo, se tuvieron diferentes reuniones para co-crear las herramientas para el trabajo con los participantes.

Las entrevistas se desarrollaron en las viviendas de cada una de las cuidadoras, acordando previamente el espacio, hora y lugar. Las cuidadoras firmaron el consentimiento informado. Las entrevistas audiograbadas fueron realizadas por los mismos investigadores de manera de asegurar la confiabilidad y confianza por parte del Instituto de Capacitación y de las cuidadoras. La pauta de entrevista se validó entre los investigadores. El estudio fue aprobado por el Comité de Ética de la Universidad CES y el Instituto.

El procesamiento de la información se inició con la codificación abierta en el software ATLAS.Ti versión 7. A través de la codificación axial se identificaron categorías y subcategorías contrastando la información emergente con aquella previamente analizada. Finalmente se realizó codificación selectiva para establecer las categorías definitivas y definir cuidadores. ${ }^{(7,8)}$

Para llegar a la teorización se exploraron los vínculos de asociación, contradicción o inclusión entre los códigos y su densidad como unidades de significación. Se realizó triangulación del análisis de la información entre investigadores principales y coinvestigadores. ${ }^{(9)}$

Durante todo el proceso investigativo se aplicaron los criterios de rigor metodológico de credibilidad, auditabilidad y transferibilidad. ${ }^{(10)}$

\section{Resultados}

La población colombiana está clasificada por niveles socioeconómicos o estratos, siendo 1 el más bajo y 6 el más alto. Las participantes pertenecían a los estratos 2 y 3 , y en su mayoría alcanzaron el nivel educativo bachiller. La experiencia que han tenido como cuidadoras ha sido amplia; en su mayoría han tenido entre 6 y 10 años de experiencia. Diez de ellas ha recibido algún tipo de capacitación para sus actividades como cuidadora, pero resaltan que gran parte de sus conocimientos también han sido adquiridos por experiencia propia. 
Tabla 1. Caracterización sociodemográfica

\begin{tabular}{llr}
\hline Variables & & Frec. \\
\hline Género & Femenino & 12 \\
Edad & $25-35$ años & 4 \\
& $35-45$ años & 6 \\
Estrato & $45-55$ años & 2 \\
& 1 & 0 \\
Nivel educativo & 2 & 6 \\
& 3 & 6 \\
Experiencia como cuidadora (años) & Primaria & 0 \\
& Bachiller & 11 \\
& Técnico & 1 \\
& De 0 a 5 años & 2 \\
& De 6 a 10 años & 4 \\
Cursos o capacitación como cuidadora & De 11 a 15 años & 3 \\
De donde ha tenido la mayor formación & De 16 a 20 años & 1 \\
como cuidadora & De 21 a 25 años & 2 \\
& No & 10 \\
& Conocimientos propios & 2 \\
& Educación con otras cuidadoras & 6 \\
\hline
\end{tabular}

Fuente: Elaboración propia (2020)

El perfil de madre sustituta es una figura llena de matices. Si bien son madres que voluntariamente deciden cuidar a un niño, reciben remuneración económica por ello. ${ }^{(11)}$ Dentro de las entrevistas mencionan en muchas oportunidades que es una labor que se realiza por amor, dado que el dinero que reciben puede, muchas veces, no ser suficiente para lo que implica cuidar un niño con discapacidad. Por otro lado, muchas de ellas no tienen una formación profesional, lo hacen a partir de la experiencia, mostrando así cómo generalmente son las mujeres quienes adoptan este tipo de responsabilidades. ${ }^{(12)}$

Históricamente, la crianza de las mujeres en diferentes culturas ha girado en torno a ser la figura de cuidado, sobre todo con aquellos más vulnerables. ${ }^{(13)}$ Además, este cuidado que se da en el entorno del hogar generalmente se asocia a una labor asistencial, sin ninguna remuneración económica, aunque en este caso sí la tienen. ${ }^{(5)}$

Una vez comenzado el proceso de codificación abierta, hay un elemento que sobresale: las rutinas diarias como categoría principal. Al continuar la codificación axial, los demás códigos emergentes entran en una relación de subordinación y pertenencia con las rutinas. Finalmente, a partir de la codificación selectiva se define el establecimiento de rutinas como categoría principal y las demás subcategorías (Figura 1). 


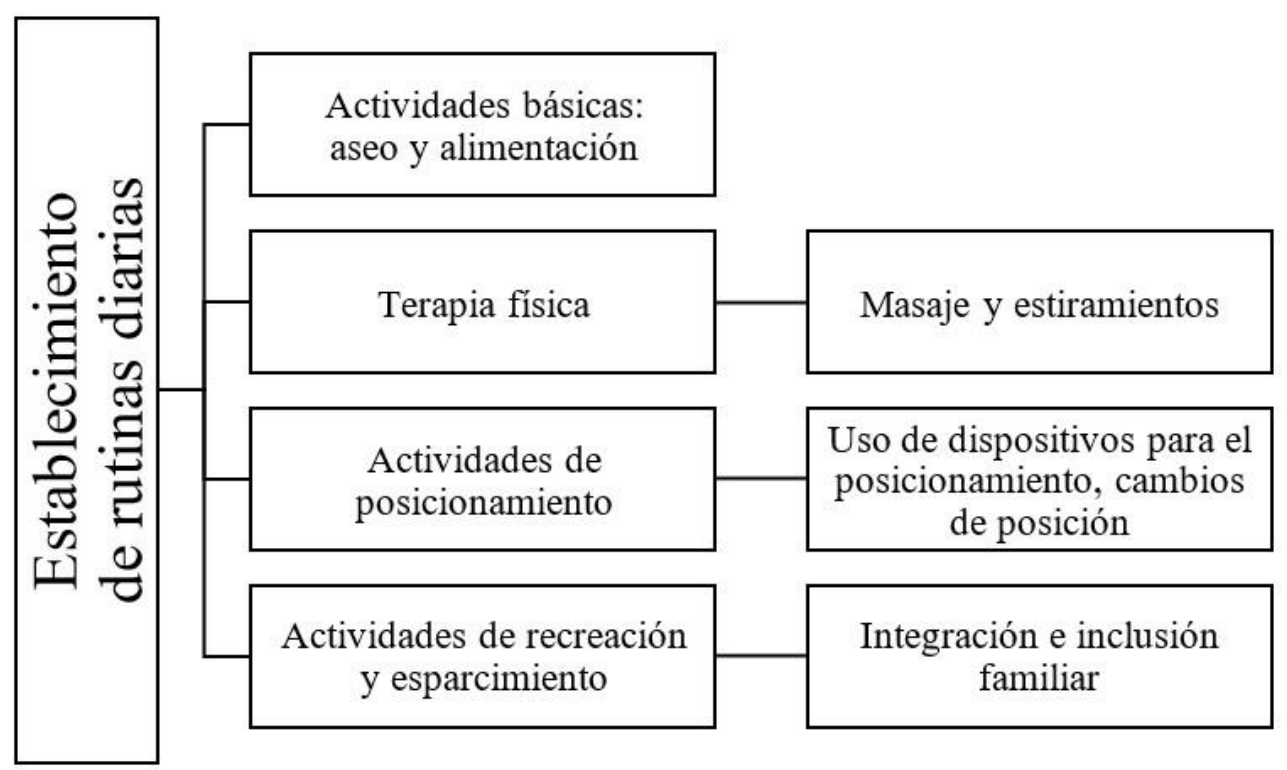

Figura 1. Categorías y subcategorías. Fuente: Elaboración propia (2020)

\section{Rutinas diarias: Aseo y alimentación}

Las rutinas son primordiales para poder realizar todas las actividades de cuidado adicional que demanda un niño con discapacidad. Estas modifican la dinámica familiar, pero es algo aceptado y vivido de manera normal por las familias, se convierte en parte de su cotidianidad, olvidando por momentos, la complejidad de la tarea que se realiza.

Un día normal es: el baño, cierto, todo lo que es el aseo personal, cepillado, corte de uñas, aseo de "gastro" [sonda de gastrostomía]. (C3)

Lo cotidiano, bañarlos, hacerles el aseo, el cepillado de dientes, su alimentación a horas... con la niña que tengo con discapacidad es más complicado por la silla de ruedas. (C9)

El aseo y la alimentación que son actividades que los niños hacen de forma autónoma. En los niños con discapacidad se convierten en actividades muy visibles, dado que requiere asistencia y su no realización conlleva a múltiples complicaciones. Además, demandan tiempo y en algunos casos conocimientos específicos para su realización. Una de las cuidadoras comparte la rutina de alimentación de uno de los niños:

Uno come de pie, paradito, y con la cabeza entre mis piernas le doy la comida, porque en otra posición es imposible... y al otro, sí me lo siento acá en la pierna y le coloco la gastro [sonda de gastrostomía] y lo mantengo sentado, porque después de que come, debe permanecer siquiera 40 minutos así bien sentado para que no broncoaspire. (C6)

\section{Terapia física}

Para la mayoría de las cuidadoras, la terapia física y el masaje cobran relevancia dadas las alteraciones del funcionamiento que tienen los niños. Estas actividades se 
convierten en elementos claves de la rutina que propenden a mantener un buen estado de salud, hacen parte de su cotidianidad y no es visto como una actividad adicional al cuidado. Los siguientes testimonios evidencian la terapia como parte de la rutina:

Lo primero que hago cuando los levanto es estirarlos, o sea, les levanto las manitos, se las tiro hacia atrás, les posiciono la columna porque como estaban acostados, entonces los siento, les hago ciertos movimientos. Ya los preparo, los baño, y después del baño hago media hora de terapia con ellos. (C6)

En la tarde le estoy haciendo otra vez los ejercicios, porque yo veo que se le están pegando mucho los deditos, entonces yo más que todo los ejercicios que le hago, es como en las manitos, porque si la siento muy tiesa. (C5)

La terapia física y todos los elementos vinculados a ella emergen como una actividad del día a día, algo que tal vez no esté presente al momento de cuidar a niños sin discapacidad. Esto se puede evidenciar en los siguientes testimonios:

Me levanto a las 6 y lo primero que hago es bañarlos; los bañamos y enseguida del baño sigue el desayuno y lo que hago pues en el baño que yo le conté ahora los ejercicios y todo eso. (C2)

Bueno, la niña se levanta diario a las 4 de la mañana, se le da pues la lechita, el "pediasure", a las 6 de la mañana ya la niña se está bañando, le hago los masajitos, las terapias, pues después del baño le echo cremita en todo el cuerpo, le hago las terapias de las manitos más que todo, ya la organizo y la acuesto un rato. (C5)

\section{Posicionamiento y actividades de recreación}

Al inicio del proyecto, la idea era determinar si las actividades de posicionamiento eran identificadas por las cuidadoras como estrategia de cuidado. Emerge de forma clara y natural cómo estas actividades son de suma importancia cuando implican actividades de participación como jugar o alimentarse:

Con un buen posicionamiento ellos ganan fuerza, gana seguridad, por ejemplo, uno de los niños cuando me lo entregaron, era con la cabeza caída siempre, entonces, él ha ido logrando adquirir mucha fuerza y aparte de eso, que al estar en un buen posicionamiento la digestión funciona mejor. (C6)

Reiteradamente en sus discursos, mencionan la importancia de adecuar los espacios donde va a estar el niño para que pueda participar de actividades como el juego y la recreación, no solo para las actividades esenciales como la alimentación:

Ya a eso de las 7:30 u 8, le doy el desayunito, ya la siento en la sillita, la pongo a jugar ya con juguetes, porque ya le gusta es sentarse en la silla. (C5)

Por otro lado, se pudo evidenciar cómo estas prácticas pueden realizarse de forma intuitiva con elementos presentes en casa o incluso ser adquiridos, si para la cuidadora es claro el beneficio que genera en la salud y el funcionamiento del niño. Lo anterior puede evidenciarse en lo siguiente: 
Sí, yo se los coloco [cojines], pues, le hago como una cuevita para sentarla, porque ella no puede con la cabeza. (C7)

No, también le pongo, todo esto [muestra cojines y cobijas] por si de pronto pierde el equilibrio y se me cae, y no se me vaya a golpear. (C2)

\section{Discusión}

El cuidado de niños con discapacidad supone la realización de múltiples actividades y diferentes prácticas específicas para la condición del niño. A pesar de complejidad de estas, terminan estando inmersas en un compilado de rutinas que se incorporan al día a día de la familia. Comprender cómo la terapia física y la alimentación pueden ser igual de importantes en el cuidado de un niño con discapacidad permite abordar mejor el quehacer de las cuidadoras y dimensionar la complejidad de su labor.

\section{Rutinas diarias}

Las rutinas pueden referirse a comportamientos ligados a actividades diarias y regulares, vinculadas a el mantenimiento de la salud. El establecimiento de rutinas en el cuidado de un niño con discapacidad permite vislumbrar el compromiso de la familia con el mantenimiento de una buena condición de salud. Unas rutinas adecuadas o inadecuadas pueden tener una relación directa en la presencia o no de la enfermedad. Además del cuidado y la salud, las rutinas son importantes por tres razones: primero, representa cuál es el principal enfoque de la familia; segundo, las rutinas están inmersas en el contexto y la cultura en la que viven las familias; tercero, son la interacción entre la persona y la dinámica de la familia. ${ }^{(14)}$

Si bien las cuidadoras establecen unas rutinas de cuidado diariamente, es sabido que cuidar un niño con discapacidad supone un cambio en estas; en este contexto, hablamos de rutinas mucho más complejas. Las actividades de cuidado inmersas en las rutinas se aprenden con la práctica diaria y al mismo tiempo implementando los consejos y recomendaciones de algunos profesionales de la salud. Sin embargo, su implementación cotidiana es una responsabilidad directa del cuidador. ${ }^{(15)}$ Entre las actividades que requieren mayor dedicación de tiempo y habilidad está la alimentación, dado que puede llegar a ser una actividad especializada y que se va perfeccionando con la experiencia. ${ }^{(16)}$ El momento de la alimentación trae consigo mayores desafíos a un niño con discapacidad y su cuidadora. Empero, con la práctica se convierte en una actividad diaria esencial, que impacta la rutina y los rituales familiares a la hora de la alimentación. ${ }^{(17)}$ Las rutinas de cuidado cobran otros significados; se convierten en rituales de cuidado que permiten mantener la salud y la integridad de la persona que se cuida, además de involucrar a toda la familia.

\section{Terapia física}

La terapia física es una actividad que se ha incorporado en las rutinas de cuidado y ocupan una gran parte de estas. Las actividades se han centrado principalmente en el masaje y los estiramientos, actividades que las cuidadoras han perfeccionado con la práctica diaria. El conocimiento de actividades "terapéuticas" por parte de las cuidadoras está inmerso en su quehacer diario. No es visto como una actividad adicional, sino que hace parte de la rutina de cuidado diario del niño. ${ }^{(18)}$ La realización sistemática de las actividades por parte de los cuidadores tiene efectos positivos en la salud de los niños. Sin embargo, Gorgon devela 
cómo esta tarea es minimizada por los profesionales de la salud, ya que los cuidadores no son tratados como actores activos en el proceso. ${ }^{(19)}$

En cuanto a las actividades que adelantan las cuidadoras en casa, es de resaltar el reconocimiento que hacen de una actividad tradicional y beneficiosa como es el masaje, que si bien es útil, demanda mucho tiempo en su realización, al igual que aquellas actividades catalogadas como terapéuticas. (20) Para Nobakht, los cuidadores deben invertir mucho tiempo en el cuidado de los niños, lo cual puede afectar a largo plazo su salud física y psicosocial. Una manera de optimizar la provisión de cuidados y el entrenamiento es recibir asesoría virtual en tiempo real. ${ }^{(21)}$ Pereira menciona que la terapia física que proveen los cuidadores en casa debe estar enfocada en buscar la independencia de los niños y sus familias. Solo de esta manera se puede disminuir la carga del cuidado y hacer actividades más eficientes para toda la familia. ${ }^{(22)}$

\section{Posicionamiento}

El adecuado posicionamiento de niños con discapacidad y alteraciones motoras es visto como parte del cuidado diario, genera bienestar al niño, previene lesiones en piel y distorsiones osteomusculares. ${ }^{(23,24)}$ Es claro que no se trata solo de los dispositivos de apoyo, también de su forma y tiempo de uso, además de las habilidades y conocimientos de los cuidadores. Entre más regulares sean estas prácticas, mayor será el beneficio para el niño. ${ }^{(25)}$

En cuanto a los dispositivos de apoyo, la evidencia muestra que, ya sea dispositivos técnicos o de fabricación casera, reportan beneficios en el funcionamiento y la participación de los niños. ${ }^{(26)}$ La participación entendida como la presencia física, la vinculación de elementos de afecto, motivación y conexión social incluye elementos comportamentales, los cuales son difíciles de evidenciar cuando se aborda el tema de la participación de niños con discapacidad en diversas actividades. En este contexto, el buen posicionamiento favorece una participación autentica. ${ }^{27,28)}$

El posicionamiento depende del conocimiento que tenga el cuidador sobre las necesidades del niño y cómo se establece una comunicación entre ambos. ${ }^{(29)}$ Lyons explora cómo elementos de posicionamiento podrían ser adaptados de forma exitosa siempre y cuando se tuviera una comunicación y una lectura acertada de las emociones de los niños. Esta labor resulta más fácil para un cuidador que conviva y se interese por el bienestar de los niños. El posicionamiento y las interacciones de comunicación dan cuenta de un compromiso genuino frente al cuidado.

Por otro lado, el cuidador puede no tener un conocimiento técnico y científico sobre el posicionamiento, pero su actuar está enmarcado en un comportamiento empático en reacción a las expresiones o sentimientos de los niños, confort o disconfort. De esta manera, la empatía depende de las características de los estímulos percibidos, el conocimiento de quien observa y su disposición a ayudar. ${ }^{(30)}$ Finalmente, Geere expone que tener acceso a dispositivos de apoyo genera beneficio no solo para el niño sino también para su cuidador. ${ }^{(31)}$ Las participantes lo evidenciaban cuando relataban cómo, una vez que dejaban al niño bien posicionado, ellas podían sentirse más tranquilas.

\section{Conclusión}

Las prácticas de cuidado realizadas por las cuidadoras del instituto develan la forma en que se realiza el cuidado de los niños con discapacidad motora de 1 a 6 años. Actividades que, a pesar de su complejidad, en cuanto a ejecución y conocimientos, se convierten en 
rutinas indispensables, enmarcadas en rituales de cuidado para cada familia. La comprensión de las prácticas y todos los elementos vinculados a ellas permiten entender el porqué de las acciones de las cuidadoras y, con ello, abordar a la población conforme a sus necesidades. Una rutina en un contexto habitual es una actividad que puede pasar desapercibida, pero es evidente que en esta población es el elemento clave en el cuidado.

Comprender la manera en que las rutinas constituyen el cuidado de un niño con discapacidad valida y legitima el quehacer de las cuidadoras, además de aportar elementos indispensables para que cuidadores profesionales e instituciones hagan ajustes en sus prácticas. Los resultados de este proyecto aportan una visión distinta en cómo debe realizarse el abordaje de esta temática con la población. Los cuidadores tienen mucho conocimiento práctico, los esfuerzos ya no deberían basarse en capacitar al cuidador, sino en generar conocimiento conjunto entre cuidadores y profesionales. Validar y legitimar las prácticas que se han construido a través de la experiencia.

Financiamiento: Proyecto financiado por la Facultad de Fisioterapia y la Dirección de Investigación e Innovación de la Universidad CES, Medellín, Colombia.

\section{Referencias bibliográficas}

1. United Nations. Convención sobre los Derechos de las Personas con Discapacidad Guía de Formación [Internet]. UN; 2014 [citado 25 de febrero de 2021]. Disponible en: https://www.un-ilibrary.org/content/books/9789210564977

2. Discapacidad Informe Mundial 2011 OMS [Internet]. [citado 25 de febrero de 2021]. Disponible http://www.codajic.org/sites/www.codajic.org/files/Discapacidad\%20Informe\%20\%2 OMundial\%202011\%200MS.pdf

3. Organización Mundial de la Salud \& United Nations Children's Fund. El desarrollo del niño en la primera infancia y la discapacidad: un documento de debate. 2013 [citado 21 de julio de 2015]. Disponible en: http://apps.who.int/iris/handle/10665/78590

4. Pérez-de la Cruz S. Parálisis cerebral infantil y el uso de sistemas de posicionamiento para el control postural: estado actual del arte. Neurología. 2017[citado 20 de febrero de 2018];32(9):610-615. Disponible en: http://linkinghub.elsevier.com/retrieve/pii/S0213485315001516

5. Martínez Marcos M, De la Cuesta Benjumea C. La experiencia del cuidado de las mujeres cuidadoras con procesos crónicos de salud de familiares dependientes. Aten Primaria [Internet]. 2016[citado 31 de marzo de 2020];48(2):77-84. Disponible en: https://linkinghub.elsevier.com/retrieve/pii/S0212656715001249

6. Hernández Sampieri R, Fernández Collado C, Pilar Baptista Lucio M. Metodología de la investigación. México: McGraw-Hill; 2014.

7. Coffey A. Encontar el sentido a los datos cualitativos [Internet]. [citado 22 de abril de 2021]. 
https://www.fceia.unr.edu.ar/geii/maestria/2014/DraSanjurjo/8mas/Amanda\%20Coffe $\mathrm{y}, \% 20$ Encontar\%20el\%20sentido\%20a\%20los\%20datos\%20cualitativos.pdf

8. Vivar CG, Arantzamendi M, López-Dicastillo O, Gordo Luis C. La Teoría Fundamentada como Metodología de Investigación Cualitativa en Enfermería. Index Enferm [Internet]. 2010[citado 29 de abril de 2021];19(4):283-288. Disponible en: https://scielo.isciii.es/scielo.php?script=sci_abstract\&pid=S1132$12962010000300011 \& \operatorname{lng}=\mathrm{es} \& \mathrm{nrm}=\mathrm{iso} \& \mathrm{t} \operatorname{lng}=\mathrm{es}$

9. Morse JM. Asuntos críticos en los métodos de investigación cualitativa [Internet]. Universidad de Antioquia; 2003 [citado 22 de abril de 2021]. Disponible en: https://dialnet.unirioja.es/servlet/libro?codigo $=663465$

10. Morse JM, Barrett M, Mayan M, Olson K, Spiers J. Verification Strategies for Establishing Reliability and Validity in Qualitative Research. Int J Qual Methods [Internet]. 2002[citado 4 de marzo de 2021];1(2):13-22. Disponible en: http://journals.sagepub.com/doi/10.1177/160940690200100202

11. Instituto Colombiano de Bienestar Familiar (ICBF). Lineamiento Técnico para las Modalidades de Vulneración o Adoptabilidad para el Restablecimiento de Derechos de Niños, Niñas y Adolescentes y Mayores de 18 años con Discapacidad, con sus Derechos Amenazados, Inobservados o Vulnerados. [Internet]. Resolución 5930 de 2010. Disponible en: https://www.icbf.gov.co/cargues/avance/docs/resolucion_icbf_5930_2010.htm\#inicio

12. Coira Fernandez G, Bailon Muñoz E. La invisibilidad de los cuidados que realizan las mujeres. Aten Primaria [Internet]. 2014[citado 31 de marzo de 2020];46(6):271-2. Disponible en: https://linkinghub.elsevier.com/retrieve/pii/S0212656714001772

13. Massé Garcia MC. La mujer y el cuidado de la vida. Comprensión histórica y perspectivas del futuro. Cuadernos de Bioética XXVIII; 2017.

14. Fiese BH. Dimensions of Family Rituals Across Two Generations: Relation to Adolescent Identity. Fam Process [Internet]. 1992[citado 26 de febrero de 2021];31(2):151-62. DOI: 10.1111/j.1545-5300.1992.00151.x

15. Denham SA. Relationships between Family Rituals, Family Routines, and Health. J Fam Nurs [Internet]. 2003[citado 25 de febrero de 2021];9(3):305-30. Disponible en: http://journals.sagepub.com/doi/10.1177/1074840703255447

16. Adams MS, Khan NZ, Begum SA, Wirz SL, Hesketh T, Pring TR. Feeding difficulties in children with cerebral palsy: low-cost caregiver training in Dhaka, Bangladesh. Child Care Health Dev [Internet]. 2012[citado 28 de abril de 2021];38(6):878-88. Disponible en: https://onlinelibrary.wiley.com/doi/abs/10.1111/j.1365-2214.2011.01327.x

17. Russell M, Jewell V, Poskey GA, Russell A. Enteral feeding and its impact on family mealtime routines for caregivers of children with cerebral palsy: A mixed method study. Aust Occup Ther J. 2018;65(1):25-34. 
18. Kruijsen-Terpstra AJA, Ketelaar M, Boeije H, Jongmans MJ, Gorter JW, Verheijden J, et al. Parents' experiences with physical and occupational therapy for their young child with cerebral palsy: a mixed studies review: Parents' experiences with their child's therapy. Child Care Health Dev [Internet]. 2014[citado 25 de febrero de 2021];40(6):787-96. DOI: 10.1111/cch.12097

19. Gorgon EJR. Caregiver-Provided Physical Therapy Home Programs for Children with Motor Delay: A Scoping Review. Phys Ther [Internet]. 2018[citado 29 de abril de 2021];98(6):480-93. Disponible

en: https://academic.oup.com/ptj/article/98/6/480/4813621

20. Weekly T, Riley B, Wichman C, Tibbits M, Weaver M. Impact of a Massage Therapy Intervention for Pediatric Palliative Care Patients and Their Family Caregivers. J Palliat Care [Internet]. 2019[citado 26 de febrero de 2021];34(3):164-7. Disponible en: http://journals.sagepub.com/doi/10.1177/0825859718810727

21. Nobakht Z, Rassafiani M, Hosseini SA, Hosseinzadeh S. A web-based daily care training to improve the quality of life of mothers of children with cerebral palsy: A randomized controlled trial. Res Dev Disabil. 2020;105:103731. DOI: 10.1016/j.ridd.2020.103731

22. Pereira A, Moreira T, Lopes S, Nunes AR, Magalhães P, Fuentes S, et al. «My Child has Cerebral Palsy»: Parental Involvement and Children's School Engagement. Front Psychol. 2016;7:1765. DOI: 10.3389/fpsyg.2016.01765

23. Angsupaisal M, Maathuis CGB, Hadders-Algra M. Adaptive seating systems in children with severe cerebral palsy across International Classification of Functioning, Disability and Health for Children and Youth version domains: a systematic review. Dev Med Child Neurol [Internet]. 2015[citado 20 de febrero de 2018];57(10):919-30. DOI: 10.1111/dmcn. 12762

24. Ágústsson A, Sveinsson P, Rodby-Bousquet E. The effect of asymmetrical limited hip flexion on seating posture, scoliosis and windswept hip distortion. Res Dev Disabil. 2017;71:18-23.

25. Sato H, Iwasaki T, Yokoyama M, Inoue T. Monitoring of body position and motion in children with severe cerebral palsy for 24 hours. Disabil Rehabil [Internet]. 2014 [citado 4 de abril de 2018];36(14):1156-60. Disponible en: http://www.tandfonline.com/doi/full/10.3109/09638288.2013.833308

26. Henderson S, Skelton H, Rosenbaum P. Assistive devices for children with functional impairments: impact on child and caregiver function. Dev Med Child Neurol [Internet]. 2008 [citado 25 de febrero de 2021];50(2):89-98. DOI: 10.1111/j.14698749.2007.02021.x

27. Alghamdi MS, Chiarello LA, Palisano RJ, McCoy SW. Understanding participation of children with cerebral palsy in family and recreational activities. Res Dev Disabil 
[Internet]. 2017 [citado 2 de marzo de 2021];69:96-104. Disponible en: https://linkinghub.elsevier.com/retrieve/pii/S0891422217301798

28. Imms C, Adair B, Keen D, Ullenhag A, Rosenbaum P, Granlund M. 'Participation': a systematic review of language, definitions, and constructs used in intervention research with children with disabilities. Dev Med Child Neurol [Internet]. 2016 [citado 3 de marzo de 2021];58(1):29-38. Disponible en: https://onlinelibrary.wiley.com/doi/abs/10.1111/dmcn.12932

29. Hotham S, Hamilton-West KE, Hutton E, King A, Abbott N. A study into the effectiveness of a postural care training programme aimed at improving knowledge, understanding and confidence in parents and school staff: Effectiveness of a postural care training programme. Child Care Health Dev [Internet]. 2017 [citado 20 de febrero de 2018];43(5):743-51. DOI: 10.1111/cch.12444

30. Lyons EA, Jones DE, Swallow VM, Chandler C. An Exploration of Comfort and Discomfort Amongst Children and Young People with Intellectual Disabilities Who Depend on Postural Management Equipment. J Appl Res Intellect Disabil [Internet]. 2017 [citado 20 de febrero de 2018];30(4):727-42. DOI: 10.1111/jar.12267

31. Geere JL, Gona J, Omondi FO, Kifalu MK, Newton CR, Hartley S. Caring for children with physical disability in Kenya: potential links between caregiving and carers' physical health. Child Care Health Dev [Internet]. 2013 [citado 28 de abril de 2021];39(3):381-92. Disponible

en: https://onlinelibrary.wiley.com/doi/abs/10.1111/j.1365-2214.2012.01398.x

Contribución de los autores: a) Concepción y diseño del trabajo, b) Adquisición de datos, c) Análisis e interpretación de datos, d) Redacción del manuscrito, e) Revisión crítica del manuscrito.

N. J. A. B. ha contribuido en a, b, c, d, e; J. M. A. H. en b, c, d, e; A. T. M. en b, c, d; A. C. P. en b, c, d; A. G. M. en b, c, d; J. F. N. L. en b, c, d; M. C. T. V. en b, c, d.

Editora científica responsable: Dra. Natalie Figueredo 